\title{
Labyrinthe
}

29 | 2008 (1)

Ce que le libéralisme promet

\section{Les espaces de la révolte}

De la discipline à la sécurité (et retour ?) : émeutes urbaines 1772/2005

\section{Déborah Cohen}

\section{(2) OpenEdition}

\section{Journals}

Édition électronique

URL : http://journals.openedition.org/labyrinthe/3463

DOI : $10.4000 /$ labyrinthe.3463

ISSN : 1950-6031

Éditeur

Hermann

Édition imprimée

Date de publication : 11 janvier 2008

Pagination : 79-90

ISBN : 9782952613163

Référence électronique

Déborah Cohen, «Les espaces de la révolte », Labyrinthe [En ligne], 29 | 2008 (1), mis en ligne le 11 janvier 2010, consulté le 22 novembre 2020. URL : http://journals.openedition.org/labyrinthe/3463 DOI : https://doi.org/10.4000/labyrinthe.3463

Propriété intellectuelle 


\title{
LES ESPACES DE LA RÉVOLTE De la discipline à la sécurité (et retour?) : émeutes urbaines 1772/2005
}

\author{
Déborah CoHEN \\ cohen.deborah@free.fr
}

Le 4 mai 1772, les jurés en charge de la communauté des luthiers de Paris et le commissaire du Châtelet J.-B. Thierry, accompagnés d'un huissier à verge et de trois sections de la garde de Paris, entrent dans l'enclos Saint-Martin-des-Champs, afin d'y confisquer les violons entreposés chez un tailleur qui contrevenait ainsi aux statuts et règlements de la communauté des luthiers. S'ensuivent sédition et révolte violente dans l'enclos'.

Le 27 octobre 2005 à Clichy-sous-Bois, la Brigade anti-criminalité poursuit B. Traoré, Z. Benna et M. Altun : âgés de 15 à 17 ans, ils reviennent d'un match de football et se sont soustraits à son contrôle. Affolés, les trois jeunes gens se réfugient dans l'enceinte d'un site EDF où deux d'entre eux trouveront la mort. C'est le point de départ d'une vague d'émeutes de plusieurs semaines dans de nombreuses cités de la banlieue française.

Quels rapports entre ces deux événements séparés par plus de deux siècles, et pourquoi les rapprocher? Le parallèle a été fait en 2005, souvent de manière incontrôlée, qualifiant les jeunes émeutiers de nouveaux sans-culottes ou les rapprochant au contraire de paysans de l'ère pré-industrielle, supposés entrer en émeute de manière éruptive et irrationnelle. Un rapprochement serein semble pourtant heuristiquement fécond en partant du fait qu'on est, dans les deux cas et par-delà le $\mathrm{XIX}^{\mathrm{e}}$ siècle, dans un cadre politique où les acteurs n'ont pas ou n'ont plus de conscience de classe et où les modèles marxistes d'analyse de

1. L'ensemble de l'analyse s'appuie sur l'information judiciaire conservée dans les archives du Châtelet sous la cote Y 9527. Toutes les citations concernant cette émeute de 1772 sont issues de ce document. 
la revendication, de la construction du groupe et de l'émeute sont donc a priori peu opératoires. Ce qui ne veut pas dire qu'une analyse en termes marxistes soit inopérante, mais qu'elle est, de toutes façons, à articuler autrement.

D'autre part, et alors qu' on est dans des modèles d'organisation du marché capitaliste très différents, des parallèles frappants existent dans le détail des formes de l'émeute. Si les émeutes de novembre 2005 restent difficiles à qualifier, tant leurs raisons et leurs objectifs paraissent brouillés, il est à tout le moins possible de les circonscrire négativement et de constater que, comme celle de l'enclos SaintMartin en 1772, il ne s'agit ni d'émeutes révolutionnaires, ni d'émeutes clairement rattachées à des revendications d'ordre constitutionnel ou politique; et qu'elles n'impliquent, au sein d'un espace perçu comme ayant une identité propre, qu'une partie des habitants. Ce qui est en jeu en 1772, c'est (dans le cadre d'un marché du travail organisé de manière corporative et surveillé par un système policier que Foucault qualifiait de disciplinaire) la possibilité de préserver des espaces particuliers pour le travail - espaces théoriquement acceptés et pourtant échappant au système ${ }^{2}$. Alors que les sources disponibles sont plus détaillées pour l'émeute d'autrefois, le rapprochement permet d'attirer le regard sur ce que le manque de données risque de nous faire rater dans l'émeute contemporaine. On voudrait ici faire l'hypothèse qu'en 2005, dans un modèle désormais libéral et sécuritaire, se joue aussi, quoique de manière voilée, un certain rapport au travail - ou à son absence.

\section{CONTRÔLE DES OBJETS, CONTRÔLE DES CONDUITES}

Avant l'émeute, avant la geste collective, il y a, dans les deux cas analysés, la rencontre entre des formes policières et des cas individuels. Du garçon tailleur qui «a chez lui un entrepôt de violons qu'il vend et débite à tout venant», nous ne saurons pas même le nom. Ce

2. Dans le système corporatif d'Ancien Régime, nul ne peut théoriquement fabriquer ou vendre en dehors des règles et des institutions du métier. En réalité, toute une part du marché échappe à cette régulation : ouvriers dits «sans qualité» parce que non enregistrés par le bureau corporatif, ou faux ouvriers, chambrelans, et ouvriers des enclaves juridiquement privilégiés comme l'est l'enclos SaintMartin. 
sur quoi se concentrent et l'enquête et la sollicitude populaire ce 4 mai 1772, c'est une caisse, qui contenait vingt-deux violons. L'émeute s'apaise une fois que la foule a constaté le départ des jurés sans leur objet : «le public étant en ce moment plus calme et paraissant satisfait de ce que la saisie n'avait pas été faite». La question du contrôle est ici médiatisée par des objets, qui sont ce par quoi le pouvoir passe pour se donner prise sur les gens et ce que ceux-ci tentent de conserver à l'écart. L'émeute de 1772 concerne un système corporatif d'organisation du travail, centré sur les modalités de fabrication des objets, et défend l'exception que constitue le lieu privilégié, contre la tentative des jurés d'y étendre leur autorité.

En revanche, à l'origine des émeutes de 2005, il n'y a pas d'objet matériel mais le heurt des corps : deux adolescents sont morts, un troisième est grièvement blessé au bout d'une course poursuite à laquelle on ne peut assigner de raison immédiate ${ }^{3}$. La volonté d'ordre a porté directement sur les conduites, réelles ou supposées. De même que l'émeute de 1772 , en se focalisant sur des objets, met en valeur une organisation du travail où ceux-ci sont au centre de l'attention, de même, l'émeute de 2005, avec sa raison apparemment absente et la prééminence des corps, invite à essayer de comprendre de quel système de contrôle pourrait relever ce mélange de présence des corps et d'absence d'objets.

\section{DES ESPACES DE RELÉGATION}

Dans les deux cas, la scène de l'émeute est celle d'espaces de relégation. Et les «faux » ouvriers de l'enclos et les habitants des cités sont l'objet d'une mise à l'écart physique et symbolique. Si les corps de métiers sont des systèmes de contrainte, ce sont aussi des systèmes de protection - dont sont exclus non seulement vagabonds et mendiants, mais aussi les ouvriers chambrelans et ouvriers privilégiés. Ceux-ci sont vite assimilés à des mendiants, puisque leur travail, non enregistré dans le système corporatif, ne peut être reconnu, et sont même exclus

3. Les sociologues ont souligné le caractère relativement immuable des scénarios de début d'émeutes urbaines, où la mort d'un des leurs met le feu aux poudres pour les habitants de cités. Voir Stéphane Beaud, Michel Pialoux, Violences urbaines, violence sociale. Genèse des nouvelles classes dangereuses, Paris, Fayard, 2003. 
de cette reconnaissance, toute instrumentale, que la fin du XVIII ${ }^{\mathrm{e}}$ siècle porte au peuple en tant qu'il constituerait, par son travail, la partie la plus utile de la nation : dans le cadre encore disciplinaire des corporations, celui qui ne travaille pas selon les normes reconnues est vite assimilé à un non-travailleur, à un inutile.

Les espaces privilégiés, échappant aux règles des métiers, participent en même temps pleinement du marché parisien encadré. Si ces ouvriers «créent un monde du travail parallèle, hors des communautés de métier, perçu comme socialement illicite, politiquement séditieux, moralement corrompu et techniquement incapable ${ }^{4}$ », ils peuvent néanmoins passer du monde des corporations à celui du travail libre et vice-versa. Les marchandises elles-mêmes circulaient entre ces deux espaces $^{5}$. Et si l'image qui leur est renvoyée est celle d'inutiles improductifs, la réalité concrète de leur investissement productif est un point d'appui pour aller contre celle-ci.

L'enclos Saint-Martin est un espace fermé, où le mur et la grille distinguent l'intérieur de l'extérieur. Disposant de leurs propres forces de surveillance, sous la forme de gardes Suisses, les habitants de l'enclos sont particulièrement jaloux de leur indépendance par rapport à la police ordinaire. L'espace de relégation est aussi un espace approprié et revendiqué. Le seul fait que les jurés et la police du Châtelet aient pu pénétrer dans l'enclos est déjà propre à mettre les habitants en furie. Au dénommé Lelièvre, sergent d'ordre commandant une section de la garde de Paris, qui lui demandait de contenir la foule, le père Procureur responsable de l'enclos aurait répondu «pourquoi y êtes-vous venu? » Et Poineau, compagnon éventailliste, explique bien au commissaire que «le peuple a commencé à se mouvoir relativement à [sa] présence et à celle de la garde». Si le peuple de l'enclos est certes assigné à cet espace marginal et son travail non validé par le système corporatif, tous visent à maintenir une liberté des corps et à retourner le stigmate en fierté : le travail non incorporé se dit libre et relève d'une exception qui se nomme aussi privilège, puisque, théoriquement, il n'est pas permis de faire saisie en ce lieu. L'autorité des jurés et de la police parisienne n'est donc pas reconnue : au commissaire, qui voulait

4. Steven L. Kaplan, «Les faux ouvriers à Paris pendant le XVIII siècle», dans La France d'Ancien Régime. Études réunies en l'honneur de Pierre Goubert, Toulouse, Privat, 1984, p. 325.

5. Voir Alain Thillay, Le Faubourg Saint-Antoine et ses «faux ouvriers». La liberté du travail à Paris aux XVII et XVIII siècles, Paris, Champ Vallon, 2002. 
discuter, «tous» auraient répondu : «tu es un j.f., nous ne te connaissons pas $^{6} \gg$. Les individus arrêtés sont en majorité de ceux que les jurés appellent «faux» ouvriers : notamment un fripier, un oiseleur, deux tailleurs d'outils, un menuisier, un faiseur de peignes, tous demeurant dans l'enclos. Leur opposition à la garde mise au service des métiers jurés s'énonce sous la forme du mépris pour celui qui ne produit rien : «viens-donc, bougre de soldat de dix-huit sols, viens-donc voleur de montre, que nous t'écrasions ». Ce qui permet à l'enclos, cet espace de relégation, de devenir ainsi le lieu d'une fière appropriation, c'est précisément qu'il est un lieu de travail et de production.

De cet espace approprié, il s'agit de disposer : le heurt avec la police se fait, à la fois symboliquement et très concrètement, autour du contrôle des grilles, que le peuple parvient à fermer. Le lieu clos est aussi un pont tendu vers l'ailleurs : d'après les déclarations de plusieurs soldats, le tailleur saisi, certain d'être défendu dans l'enclos, peut narguer la garde en revenant après sa fuite. Comme le garde s'opposait à sa rentrée, «il a dit qu'il était prêt de gager six francs qu'il rentrerait malgré la garde et que la garde ne sortirait pas». S'amusant, il aurait encore déclaré : «je sortirai, je rentrerai et vous n'en sortirez pas ». L'enclos devient un piège pour la garde et le lieu d'un pouvoir relatif pour les travailleurs non incorporés. On voit que le cadre disciplinaire définissait les individus et leur assignait un lieu dans l'en dedans ou l'en-dehors, mais il ne parvenait pas à les consommer entièrement, à priver complètement ceux qu'il excluait de toute possibilité de résister à partir d'un point extérieur à la discipline. La discipline crée ses propres espaces de contradiction et les points d'appui à retourner contre elle.

Quant aux habitants des cités, surtout si leur visage ou leur nom indique un parcours familial d'immigration, ils sont souvent objets de mépris, voire d'injures et de violence. Après bien des discours simplificateurs sur l'exclusion, on a montré la complexité de la situation des jeunes des cités de banlieue : ni «dehors» parce qu'ils partagent bien souvent, avec l'ensemble des citoyens français, et la nationalité et un certain nombre de valeurs, parce que la cité n'est pas un ghetto homogène dont on ne sort jamais ${ }^{7}, \mathrm{ni}$ «dedans » parce que socialement leur

6. « Connaître » quelqu'un, dans la langue du XVIII' siècle, c'est avouer son autorité.

7. Voir Loïc Wacquant, Parias urbains. Ghetto, banlieue, État, trad. Sébastien Chauvin, Paris, La Découverte, 2006. 
situation est souvent plus précaire ${ }^{8}$. Néanmoins, à regarder le rapport à l'espace que révèle l'émeute, le statut de ces espaces de relégation que sont les cités révèle des différences significatives par rapport à celui offert par l'enclos Saint-Martin.

En régime libéral, même l'espace de relégation est un espace ouvert : point de mur qui apparenterait les cités à l'enclos SaintMartin. Mais si les ouvriers de l'enclos traversaient cette frontière matérielle avec facilité, et s'en vantaient, transformant le stigmate en choix et l'exclusion en possession d'un espace propre, les cités, elles, sont plus subtilement séparées, par le simple jeu du marché conduisant notamment à une offre de transports réduite. Cette séparation ne semble pas pour autant permettre une appropriation de l'espace. Il ne semble pas y avoir à ce jour de construction forte d'une «identité de cité», qui retournerait le stigmate en fierté ${ }^{9}$.

Certes, au quotidien mais à la marge, de petits groupes semblent mimer une prise de possession du quartier : ils «tiennent les murs», occupent les halls d'immeuble ou se postent devant le centre commercial, provoquant ceux qu'ils perçoivent comme plus «installés». Pour autant, comme elle ne s'ancre justement dans aucune possession, dans aucune production, cette appropriation du quartier paraît illusoire, et pour la majorité des habitants et pour les minorités provocantes qui en construisent l'apparence. Si le tailleur de 1772 se jouait de la frontière entre l'enclos et l'extérieur et pouvait trouver refuge derrière les grilles, les trois jeunes garçons morts en octobre 2005 semblent montrer, par leur fuite, qu'il n'y avait pas, à l'intérieur de la cité, de lieu approprié qui pût constituer un refuge. D'où leur passage derrière un mur qui, tragiquement, loin d'ouvrir sur le familier, ouvre sur l'inconnu du danger et d'un transformateur EDF.

Les deux émeutes, à plus de deux siècles de distance, partagent certains traits : l'émeute de 2005 ressemble fort à celle de 1772 dans les formes de refus de l'intrusion policière, mais aussi dans une certaine retenue de la violence contre les personnes. Les menaces, telles qu'elles sont rapportées par les soldats de la garde, n'ont assurément pas

8. Voir Robert Castel, La Discrimination négative. Citoyens ou indigènes, Paris, Seuil, 2007.

9. Voir Thomas Sauvadet, «Le sentiment d'insécurité du "dealer de cité" ", Le Temps de l'histoire. Sociétés et jeunesses en difficulté (http://sejed.revues.org/document122.html [consulté le 5 janvier 2008]). 
manqué en 1772 : un compagnon menuisier accusé de s'être porté à la tête de l'émeute, aurait dit «vous ne sortirez pas d'ici en vie», ou encore «mâtin, tu ne sortiras d'ici qu'en pièces et en morceaux». Mais il n'y aura pas un mort. De même en 2005, on a souligné l'absence de montée aux extrêmes de la violence. Néanmoins, les deux émeutes diffèrent dans la mesure où, en 2005 , la violence ne s'est pas exercée seulement contre des forces de police perçues comme extérieures et que l'on tenterait de repousser au-delà d'un espace de l'entre-soi, mais aussi contre l'espace de la cité elle-même. Les émeutiers auraient détruit «leurs » écoles, «leurs» gymnases. À moins de ne voir dans ces jeunes émeutiers que la figure du seul désespoir en mouvement et, en renvoyant leur geste à une pure autodestruction, lui retirer tout sens possible, la question ne peut pas ne pas se poser de savoir jusqu'à quel point ces bâtiments publics étaient «leurs», avaient été appropriés, et donc jusqu'à quel point, en régime libéral, le contrôle aboutit à la création d'un en-dedans et d'un en-dehors. Les propos d'un jeune diplômé sans emploi emprisonné pour avoir lancé des boules de pétanque sur les CRS illustrent bien ce point : «il n'y a rien d'autodestructeur à mettre le feu là où nous habitons, si cela permet d'arracher par la force au contrôle et à la pacification des zones où leur droit et leur justice de merde cessent enfin de s'exercer, ne serait-ce que pendant quelques heures ${ }^{10} »$. Si la discipline créait un espace conforme et un espace de l'autre qui pouvait être approprié, il ne paraît pas évident qu'il en soit de même en régime libéral, où les formes du contrôle se font sur le mode de ce que Foucault appelait sécurité.

Pourquoi l'école, la «maison des Jeunes et de la Culture» et d'autres bâtiments publics sont-ils des cibles et non des lieux de refuge, des symboles à détruire et non des signes d'espoir? Il faut sans doute ici prendre l'hypothèse foucaldienne au sérieux : en régime libéral, le gouvernement n'intervient plus en prise directe sur les gens et sur les choses, à partir d'un réseau de dispositifs, d'appareils institutionnalisés (telles que pouvaient l'être les corporations par exemple), mais les mécanismes de maîtrise se font inhérents au champ social, en comprenant les moyens d'information et d'éducation, les systèmes de protection de santé... Autant de structures qui encadrent la vie et la

10. «Fils de la République? Alors, nique ta mère !». Blogs de détenus de la prison de..., janvier 2006, dans Jean-Pierre Garnier, «Une violence éminemment contemporaine. L'espace public urbain comme scène "post-historique"», Espaces et sociétés, vol. 128-129, n 1-2, 2007, p. 68. 
pensée de manière à empêcher d'éventuels blocages dans le fonctionnement du libre marché. Dans la cité, donc, pas de mur, pas de grille, pas d'objet non plus dont la forme et les modes de fabrication seraient soumis à contrôle; néanmoins, c'est bien en replaçant le marché à l'horizon de tout cela que l'on peut y comprendre quelque chose. La violence s'exerce contre les symboles et les représentants d'un ordre social dont on se sent exclu ${ }^{11}$. Ainsi l'école peut-elle être pensée en lien avec les besoins du marché sur lequel elle ouvre théoriquement. De plus, tandis qu'elle formule la promesse d'une promotion sociale, elle ne peut seule en accomplir la possibilité. En ce sens, elle ne peut être pleinement le lieu d'investissement du moi, elle n'est pas un lieu propre, à l'écart, comme pouvaient l'être les boutiques de l'enclos Saint-Martin; elle est comme une propédeutique au marché, et plus spécifiquement au marché du travail. Or, de ce marché, les habitants des cités se savent particulièrement exclus. C'est largement de cette exclusion-là que témoignent les attaques contre les bâtiments publics et municipaux. Les jeunes émeutiers expriment une rage non pas seulement contre ce qui est, mais contre le visage d'un avenir qui leur est présenté au miroir de leurs aînés et de leurs pères. S'en prendre à l'école, c'est manifester la compréhension que celle-ci n'est pas un lieu d'appropriation possible, mais le lieu où se prépare pour eux l'exclusion du marché du travail ${ }^{12}$. Elle se prépare de deux façons, l'une touchant tous les enfants des classes populaires des cités, l'autre particulière aux descendants d'immigrés.

Il faut rappeler que les émeutes de 2005 n'ont pas été, en dépit des vociférations explicitement racistes de certains, le fait exclusif des enfants issus de l'immigration. Dans les régions de l'hexagone les plus sinistrées socialement, ils étaient même en minorité ${ }^{13}$. Depuis les années 1980, on a assisté à un «durcissement de la compétition scolaire au détriment des familles les moins dotées en ressources culturelles ${ }^{14} \gg$. Par le biais des titres scolaires, ou de leur absence, l'exclusion se donne

\footnotetext{
11. Emmanuel Renault, L'Expérience de l'injustice. Reconnaissance et clinique de l'injustice, Paris, La Découverte, 2004, p. 108-117 notamment.

12. Voir Stéphane Beaud, «L'insertion professionnelle en question», dans Comprendre les violences urbaines, Regards sur l'actualité, n 319, Paris, La Documentation française, mars 2006.

13. Gérard Mauger, L'Émeute de novembre 2005 ; une révolte protopolitique, Bellecombe-en-Bauges, Éditions du croquant, 2006, p. 69. Voir le compte rendu par Arnault Skornicki, dans Labyrinthe, $\mathrm{n}^{\circ} 28,2007$ (3), p. 151-156.

14. Stéphane Beaud et Olivier Masclet, «Des "marcheurs" de 1983 aux "émeutiers" de 2005. Deux générations sociales d'enfants d'immigrés », Annales HSS, n 4, juillet-août 2006, p. 827.
} 
une forme présentable : si les jeunes des cités n'ont pas de travail, ce serait parce qu'ils ont été plus nombreux à connaître l'échec scolaire. $26 \%$ des jeunes gens déférés au juge en Seine-Saint-Denis étaient déscolarisés, une majorité était inscrite dans l'enseignement professionnel - dont les diplômes sont dévalués, et connus comme tels par ceux qui les préparent. Jusqu'à un certain point, le diplôme permet une mise en ordre endossée par ceux-là mêmes qui la subissent. Les «faux» ouvriers placés hors du système corporatif, n'adhéraient pas à ce système (on a vu leur refus de reconnaître le commissaire), ils pouvaient développer des contre-valeurs et une autre idée du travail. La révolte se faisait dans les failles du système, dans les à-côtés mal contrôlés qu'il essayait de récupérer. En revanche, les jeunes issus des couches populaires contemporaines les plus défavorisées adhèrent la plupart du temps au système du diplôme qui marquera pourtant leur exclusion. Le moment de l'émeute marque alors le refus momentané de participer à cette illusion : il exhibe le diplôme, et l'école qui le délivre, comme lieux de mise en ordre et de contrôle. L'émeute ne se fait pas dans les failles d'un système; elle est une prise de distance lucide, mais forcément précaire et temporaire, par rapport aux pratiques et aux illusions quotidiennes.

\section{LES MOTS DE LA RÉVOLTE}

Les émeutiers de 1772 ne reconnaissent pas l'ordre corporatif. Ils ont construit une sorte de contre-monde reposant sur leur exclusion même, ou leurs privilèges. Ils peuvent s'appuyer sur les normes de leur travail pour revendiquer le départ de la police. Marraux, sergent de la garde de Paris, dépose ainsi qu'un individu «est venu pour converser avec lui, lui disant qu'il n'était pas permis de saisir dans des endroits privilégiés». Le sergent reconnaît qu'il y a eu tentative de dialogue, s'appuyant sur la certitude d'un droit. Mais «il lui a dit de se retirer sans vouloir l'écouter» et ordre a été donné de l'arrêter. Même si la parole populaire n'est pas entendue, dans cet espace qui se pose comme certain du droit de sa différence, elle existe. Pour justifier leur intervention, les habitants de l'enclos ont dit à haute voix : «cela nous regarde, où sont donc nos privilèges, nous payons les loyers assez chers pour que l'on nous soutiennent». Sont donc clairement posées dans le discours les deux instances qui se matérialisaient dans l'espace : l'in- 
térieur avec les travailleurs exclus du système corporatif, et l'extérieur, représentant l'ordre corporatif et disciplinaire qu'ils ne reconnaissent pas. Deux façons d'envisager le travail se font face; deux façons d'envisager une même réalité trouvent des points d'appuis différents.

Pour autant, les élites de l'époque ne reconnaissent aucune capacité politique à ces travailleurs et les mots de leur révolte ne peuvent être ni récoltés, ni entendus comme autre chose que du bruit. Sur la scène policière, lors des arrestations et des interrogatoires qui s'ensuivent, l'argumentation des prévenus n'est pas réellement prise en compte. Toute parole populaire est ramenée à ce qu' on la veut être d'avance, le frivole, le scatologique et l'insulte.

De la révolte de 2005 il a été dit qu'elle ne s'était pas mise en mots. En se souvenant de l'effacement de la parole politique populaire au $\mathrm{XVIII}^{\mathrm{e}}$ siècle, on ne peut négliger l'hypothèse que, de ce qui aurait pu être dit, rien ou peu aurait été entendu et enregistré par les médias : la manière dont la revendication de démission du ministre de l'Intérieur a été renvoyée dans le domaine du si peu sérieux qu'il en devenait comme non existant pourrait en être l'indice. Au-delà de cette demande, les revendications précises ont sans doute été peu nombreuses. La jeunesse des participants ne compte pas pour rien dans ce quasi mutisme. Mais, plus profondément, il semble que ce silence tienne aussi aux formes du pouvoir s'exerçant sur les cités.

Les ouvriers chambrelans s'appuient sur une contre-culture et, derrière les grilles de l'enclos, ont forgé un lieu propre; en 2005, pour les habitants des cités, les formes spatiales ne permettent pas de poser une pure extériorité à laquelle s'opposerait un intérieur appropriable. De même, si le discours revendicatif est rare, on peut le comprendre sur fond d'absence de lieu d'investissement pour le soi et de mots qui iraient avec. Comment revendiquer sans être dans une sorte d'extériorité face au pouvoir contesté et sans avoir quelque chose à lui opposer, sans avoir, pour parler d'un objet commun (en l'occurrence ici, le travail), un autre registre de discours? Or, un des rares slogans qui nous est parvenu des nuits de novembre montre l'homogénéité entre le discours reconnu par le pouvoir et le discours des cités : «Liberté, égalité, fraternité, mais pas dans les cités ${ }^{15}$.» Aux jurés qui leur parlaient

15. Robert Castel, «La discrimination négative. Le déficit de citoyenneté des jeunes de banlieue», Annales HSS, $\mathrm{n}^{\circ}$ 4, juillet-août 2006, p. 788. 
discipline, les ouvriers de l'enclos Saint-Martin répondaient liberté. En 2005 à Clichy, il n'y a pas de contre-monde : la liberté elle-même est investie par le pouvoir; ne reste que le regard embué porté sur des valeurs intériorisées, sur un monde désiré auquel on n'a pas accès. Reste alors seulement la mise en valeur d'une absence. Tandis que les faux ouvriers de 1772 opposaient la positivité de leur monde à celui des jurés, les émeutiers de 2005 n'ont que leur manque à exhiber. Le manque, puisque ce qui est en question, ce ne sont pas les formes du travail, mais son absence même.

\section{VERS UN RETOUR DU DISCIPLINAIRE?}

La question centrale était clairement celle du manque d'avenir, celle de l'absence du travail qui permet de s'inscrire dans ce que Robert Castel a appelé «une société de semblables», où tous ont des ressources communes et des droits communs, où tous peuvent alors être à la fois indépendants, libres, et liés par une interdépendance ${ }^{16}$. La question était donc celle d'un certain (dys)fonctionnement du libre marché. Ce que manifestaient ces émeutes, c'est que les mécanismes de l'économie libérale ne suffisent plus à assurer ni la sécurité sociale pour tous, ni la sécurité civile. Pourtant, ce n'est pas ainsi que la question a été traitée par le gouvernement (et une partie de la gauche). En instaurant l'état d'urgence et en décrétant le couvre-feu dans les cités, le gouvernement a eu recours à une loi, votée en 1955 pour s'appliquer aux indigènes algériens, et qui, en son article 5 , «donne pouvoir au préfet [...] d'interdire la circulation des personnes ou des véhicules dans les lieux et aux heures fixés par arrêté» et «d'instituer, par arrêté, des zones de protection ou de sécurité où le séjour des personnes est réglementé», c'est-à-dire qu'elle en revient à un modèle territorial. Une population donnée est définie par son appartenance à un espace. Soudain, l'espace théoriquement ouvert de la cité se met à ressembler à l'enclos Saint-Martin, comme si des barrières s'étaient dressées. Derrière ces barrières, on isole une population d' «inutiles». Ces chômeurs, ces rejetés du marché du travail (par manque de qualification ou par racisme du marché), semblent désormais aussi inutiles que

16. Robert Castel, L'Insécurité sociale. Qu'est-ce qu'être protégé ?, Paris, Seuil, 2003. 
les chambrelans du XVIII ${ }^{e}$ siècle, aussi extérieurs à la production que ceux que n'incluait pas le système des corporations. Loin de fonctionner selon son concept et sans entraves, le libéralisme se dissoudrait donc ici en disciplinaire, par reconstitution d'espaces fragmentés et d'enfermement de ceux qui paraissent menacer la sécurité du système.

Contrairement aux chambrelans installés derrière l'enclos de SaintMartin, les habitants des cités n'ont, pour l'instant, pas de point d'ancrage pour se forger une identité permettant de résister à cette imposition disciplinaire. À moins qu'ils ne se voient conduits à la trouver dans une revendication identitaire ou religieuse - dont ils sont loin pour le moment, mais vers laquelle les conduit le mur disciplinaire qu'on érige autour d'eux? 